\title{
Current Status of Biology, Bioengineering, and Therapeutic Potential of Stem Cells
}

\author{
Hyuk-Jin Cha ${ }^{1}$ and Eun Sook Hwang ${ }^{2}$ \\ ${ }^{1}$ College of Natural Sciences, Department of Life Science, Sogang University, Seoul 121-742, Korea and \\ ${ }^{2}$ College of Pharmacy, Division of Life and Pharmaceutical Sciences and Center for Cell Signaling \& \\ Drug Discovery Research, Ewha Womans University, Seoul 120-750, Korea
}

For several decades, patients with obstinate diseases such as leukemia, sickle cell anemia, and immune disorders have been treated with bone marrow transplant, of which therapeutic potential results from the regeneration capability of hematopoietic stem cells (HSCs). The dramatic regeneration potential of HSCs has been proven in tens of thousands cases since it is firstly performed in 1959 (Orkin and Zon, 2008). The regeneration potential of non-HSC stem cells derived from various tissues such as brain, bone marrow, peripheral blood, blood vessels, skeletal muscle, skin, teeth, heart, gut, liver, ovarian epithelium, and testis (Laird et al., 2008; Murry and Keller, 2008) has drawn much attention so that numerous studies have been performed in order to apply such stem cells' properties to therapeutic purpose. In late 1960s, bone marrow stromal stem cells (also called mesenchymal stem cells: MSCs) were discovered to co-exist with HSCs in adult bone marrow (Friedenstein et al., 1968). These non-HSC stem cells in bone marrow, of which known function was to support HSCs, were later demonstrated to be capable of multi-differentiating into various mesenchymal tissues such as bone, cartilage, fat, muscle, and fibrous connective tissue (Pittenger et al., 1999). Moreover, adult brain, which has been believed to be one of the terminally differentiated organs so that no regeneration was capable once it is damaged, has been shown to contain stem cells that generate nerve cells, non-neuronal astrocytes, and oligodendrocytes (Lendahl et al., 1990). This outstanding work triggered search for adult stem cells in the other terminally differentiated tissues culminating in the discovery of various types of adult stem cells. Epithelial

Correspondence to: Eun Sook Hwang, College of Pharmacy and Division of Life and Pharmaceutical Sciences, Ewha Womans University, Science Bldg C206, Seoul 120-750, Korea

Tel: 82-2-3277-4369, Fax: 82-2-3277-3760

E-mail: eshwang@ewha.ac.kr stem cells (Barker et al., 2007), epidermal and follicular stem cells (Alonso and Fuchs, 2003; Lavker et al., 2003), endothelial progenitor/stem cells (EPCs) (Stump et al., 1963; Asahara et al., 1999; Bhattacharya et al., 2000; Gehling et al., 2000), cardiac stem cells (Bearzi et al., 2007) and so on, have been newly identified. Their functional characterization and the possible therapeutic potentials have been being extensively studied. On the other hand, with the technical advance of molecular biology, many outstanding researchers even achieved to obtain the artificial types of stem cells. Differentiation destiny of adult stem cells is limited to the specialized cell types of the tissue in which they reside. MSCs can only differentiate into the mesenchymal cell types as mentioned above. Nevertheless, recent studies have reported "transdifferentiation" that certain adult stem cells are able to differentiate into different cell types other than the predicted cell types by a set of specialized differentiation techniques. Furthermore, fully differentiated normal cells can be even artificially converted to embryoniclike stem cells through 'reprogramming' by genetic introduction of a set of transcription factors producing 'induced pluripotent stem cells (iPSCs)' (Okita et al., 2007; Yu et al., 2007). As such reprogramming technique enables the patient somatic cells such as skin fibroblasts to form their own pluripotent stem cells, which was impossible by then, cell therapy based on iPSCs derived from patient's own cells has been believed to overcome the current technical barriers such as ethical issues and allosteric immune rejection. Considering that the recent trials of a human embryonic stem cells (hESCs)-derived cell therapy were discontinued due to many serious barriers to the clinical trials of hESCs, including ethical issues, tumor formation, and immune rejection (Majumder and Cohen, 2009), generation of iPSCs would be one of the most critical scientific achievements in the cell-based regenerative medicine. 
However, several unexpected drawbacks of iPSCs have been reported to undermine the therapeutic potential of iPSCs-based cell therapy. Cells derived from iPSCs have been demonstrated to be immunogenic to the own body (Okita et al., 2011). In addition, unlike the embryonic stem cells (ESCs), differentiation capability of iPSCs to a certain cell type is much favored to their somatic tissue of origin due to the epigenetic memory of the donor tissue. This may severely dampen the efforts for the directed differentiation. Thus, the possible therapeutic applications of iPSCs remain unresolved because of various technical challenges so far (Okita et al., 2011).

Adult stem cells-based cell therapy was thought to be free from most of the risk that pluripotent stem cells-based therapy has shown (Condic and Rao, 2010). Therefore, more than $70 \%$ of stem cells-based research was focused on the experimental and clinical applications of adult stem cells. Adult skin stem cells have been used to treat severe burns over past 20 years (Chen et al., 2009). Successful transplantation of adult neural stem cells into the brain of Parkinson's disease patients restored control of movement by compensating for the loss of one specific type of nerve cells that control movement (Gaillard and Jaber, 2011). Furthermore, transplantation of insulin-producing pancreatic stem cell together with blood transplants substituted for the repeated insulin injections in type I diabetes and even replenished healthy white blood cells to patients with malfunctioning immune systems (Longoni and Mosca, 2011). However, adult stem cellsbased cell therapy is also not free from the technical challenge. The source of adult stem cells is too limited to supply sufficient amount for cell therapy because those should be isolated from each individual donor and then expanded by in vitro culture. Moreover, premature senescence and the following loss of stemness during in vitro culture make the stable production of adult stem cells even more difficult (Lee et al., 2009). Unlike the conventional assumption that adult stem cells are free from the accumulated genetic mutation due to the existence of 'immortal DNA strand' (referred as 'immortal strand hypothesis') (Karpowicz et al., 2005; Rando, 2007), genetic alteration of adult stem cells during continuous in vitro culture was described (Sareen et al., 2009; Tarte et al., 2010). Thus, it is clear that there is a remote gap between the expectations of risk-free clinical application of stem cells including both pluripotent stem cells (hESCs and iPSCS) and adult stem cells and the current scientific circumstance of stem cells biology. The researchers of diverse fields of stem cells are currently dedicating their efforts to resolve the technical challenges, owing to the clear therapeutic advantages of stem cellsbased therapy as compared with the conventional approach in the aspect of the regenerative medicine.

This special issue provides the understanding of current status of stem cells-based cell therapy from basic biology to therapeutic applications. Nine outstanding review articles cover the topics of first, the biology and therapeutic potential of MSCs and EPCs in areas of tissue repair, immune modulation, diabetic vascularization, and tumor development, second, the development of iPSCs and amniotic fluid-derived stem cells (AFSCs) and their applications in disease treatment, and third, chemical approaches for drug development in stem cell research.

The first review by Hong et al. starts from basic cell biology of MSCs and delineates the molecular mechanism of reparative stem cell function of MSCs in damaged tissues. The unexpected properties and clinical perspectives of MSCs such as immune modulation and 'homing' action to the area of tumor are also discussed (Hong et al., 2012). Continuously, the second review by Yi and Song excavates the suppressive function of MSCs in the proliferation of T and B lymphocytes, the maturation and terminal differentiation of dendritic cells and B lymphocytes, and the immune reactivity of natural killer cells and macrophages. The article further describes the mechanism of immunomodulatory effects of MSCs and introduces examples of animal and clinical uses of MSCs' immune-suppressive function in the treatment of immune-related diseases including graft-versus-host disease, rheumatoid arthritis, experimental autoimmune encephalomyelitis, sepsis, acute pancreatitis, and multiple sclerosis (Yi and Song, 2012). Next, EPCs, a subpopulation of bone marrow stem cells that are critical for neovascularization and vascular homeostasis are overviewed. Kim et al. specifically discusses the functional defect of innate EPCs under diabetic conditions and suggests the mechanism for diabetic dysfunction of EPCs, which may result in the defective vascular function in the peripheral region and consequent diabetic vascular diseases (Kim et al., 2012). This review delineates an importance of the regulation of stemness or regeneration potential under a variety of stress condition and the link to human diseases. Lee et al. summarizes the possible link between a loss of stemness of stem cells and aging-related diseases such as cancer and degenerative diseases. The review describes the mal-differentiation of stem cells upon oncogenic challenges and the different responses important to determine either onset of cancer or degenerative diseases during aging. Moreover, the article indicates that stem cells are more susceptible to the oncogenic challenges than the other 
differentiated counterpart, proposing that stem cells would be a 'cell of origin' of a few cancers (Lee et al., 2012).

Novel approaches to generate iPSCs and AFSCs are introduced by the following reviews. Generation of patient-specific iPSCs, progression of iPSCs technology, and practical applications are introduced by Song et al. (2012). In addition, Zhou et al. reviews the generation of hepatocyte-like cells from iPSCs and their clinical implications in understanding pathogenesis of hepatitis B virus (HBV) and hepatitis $\mathrm{C}$ virus (HCV) and providing screening systems for treating HBV or HCV infection-mediated cirrhosis and hepatic tumor (Zhou et al., 2012). Joo et al. introduces the multipotent AFSCs, which were recently isolated from amniotic fluid and discusses characterization of stemness of AFSCs. The practical application of AFSCs as regenerative medicine is also discussed (Joo et al., 2012).

The last part is composed of chemical approaches for drug development in stem cell research. Choi and Nam offer the high throughput screening tool to screen small molecules which promote self-renewal, direct cell lineage specification, and reprogram the genetic expression of adult stem cells (Choi and Nam, 2012). Shin et al. broadly overviews most of current available engineering methods that are used in cell-based researches for chemical screening for stem cell drug development (Shin et al., 2012).

In summary, valuable reviews in this special issue present critical information on the advances in stem cell research fields covering from stem cells biology to current biomedical and chemical approaches to develop regenerative medicine and stem cell-based therapeutics for treating incurable degenerative human diseases. We expect that this special issue provides insights of stem cells research and their therapeutic opportunities and contributes to the advancement of the relevant research areas.

\section{REFERENCES}

Alonso, L. and Fuchs, E., Stem cells of the skin epithelium. Proc. Natl. Acad. Sci. U. S. A., 100 Suppl 1, 11830-11835 (2003).

Asahara, T., Masuda, H., Takahashi, T., Kalka, C., Pastore, C., Silver, M., Kearne, M., Magner, M., and Isner, J. M., Bone marrow origin of endothelial progenitor cells responsible for postnatal vasculogenesis in physiological and pathological neovascularization. Circ. Res., 85, 221-228 (1999).

Barker, N., van Es, J. H., Kuipers, J., Kujala, P., van den Born, M., Cozijnsen, M., Haegebarth, A., Korving, J., Begthel,
H., Peters, P. J., and Clevers, H., Identification of stem cells in small intestine and colon by marker gene Lgr5. Nature, 449, 1003-1007 (2007).

Bearzi, C., Rota, M., Hosoda, T., Tillmanns, J., Nascimbene, A., De Angelis, A., Yasuzawa-Amano, S., Trofimova, I., Siggins, R. W., Lecapitaine, N., Cascapera, S., Beltrami, A. P., D'alessandro, D. A., Zias, E., Quaini, F., Urbanek, K., Michler, R. E., Bolli, R., Kajstura, J., Leri, A., and Anversa, P., Human cardiac stem cells. Proc. Natl. Acad. Sci. U. S. A., 104, 14068-14073 (2007).

Bhattacharya, B., Puri, S., and Puri, R. K., A review of gene expression profiling of human embryonic stem cell lines and their differentiated progeny. Curr. Stem Cell Res. Ther., 4, 98-106 (2009).

Bhattacharya, V., McSweeney, P. A., Shi, Q., Bruno, B., Ishida, A., Nash, R., Storb, R. F., Sauvage, L. R., Hammond, W. P., and $\mathrm{Wu}, \mathrm{M}$. H., Enhanced endothelialization and microvessel formation in polyester grafts seeded with CD34(+) bone marrow cells. Blood, 95, 581-585 (2000).

Chen, M., Przyborowski, M., and Berthiaume, F., Stem cells for skin tissue engineering and wound healing. Crit. Rev. Biomed. Eng., 37, 399-421 (2009).

Choi, Y. and Nam, T.-g., Chemical biology in stem cell research. Arch. Pharm. Res., 35, 281-297 (2012).

Condic, M. L. and Rao, M., Alternative sources of pluripotent stem cells: ethical and scientific issues revisited. Stem Cells Dev., 19, 1121-1129 (2010).

Friedenstein, A. J., Petrakova, K. V., Kurolesova, A. I., and Frolova, G. P., Heterotopic of bone marrow. Analysis of precursor cells for osteogenic and hematopoietic tissues. Transplantation, 6, 230-247 (1968).

Gaillard, A. and Jaber, M., Rewiring the brain with cell transplantation in Parkinson's disease. Trends Neurosci., 34, 124-133 (2011).

Gehling, U. M., Ergun, S., Schumacher, U., Wagener, C., Pantel, K., Otte, M., Schuch, G., Schafhausen, P., Mende, T., Kilic, N., Kluge, K., Schafer, B., Hossfeld, D. K., and Fiedler, W., In vitro differentiation of endothelial cells from AC133-positive progenitor cells. Blood, 95, 3106$3112(2000)$.

Hanahan, D. and Weinberg, R. A., Hallmarks of cancer: the next generation. Cell, 144, 646-674 (2011).

Hipp, J. and Atala, A., Sources of stem cells for regenerative medicine. Stem Cell Rev., 4, 3-11 (2008).

Hong, H. S., Kim, Y. H., and Son, Y., Perspectives on mesenchymal stem cells: tissue repair, immune modulation, and tumor homing. Arch. Pharm. Res., 35, 201-211 (2012).

Joo, S., Ko, I. K., Atala, A., Yoo, J. J., and Lee, S. J., Amniotic fluid-derived stem cells in regenerative medicine research. Arch. Pharm. Res., 35, 271-280 (2012).

Karpowicz, P., Morshead, C., Kam, A., Jervis, E., Ramunas, J., Cheng, V., and van der Kooy, D., Support for the immortal strand hypothesis: neural stem cells partition DNA asymmetrically in vitro. J. Cell Biol., 170, 721-732 (2005).

Karussis, D., Kassis, I., Kurkalli, B. G., and Slavin, S., Immunomodulation and neuroprotection with mesenchymal 
bone marrow stem cells (MSCs): a proposed treatment for multiple sclerosis and other neuroimmunological/neurodegenerative diseases. J. Neurol. Sci., 265, 131-135 (2008).

Kim, K.-A., Shin, Y.-J., Kim, J.-H., Lee, H., Noh, S.-Y., Jang, S.-H., and Bae, O.-N., Dysfunction of endothelial progenitor cells under diabetic conditions and its underlying mechanisms. Arch. Pharm. Res., 35, 223-234 (2012).

Laird, D. J., von Andrian, U. H., and Wagers, A. J., Stem cell trafficking in tissue development, growth, and disease. Cell, 132, 612-630 (2008).

Lavker, R. M. and Sun, T. T., Heterogeneity in epidermal basal keratinocytes: morphological and functional correlations. Science, 215, 1239-1241 (1982).

Lavker, R. M., Sun, T. T., Oshima, H., Barrandon, Y., Akiyama, M., Ferraris, C., Chevalier, G., Favier, B., Jahoda, C. A., Dhouailly, D., Panteleyev, A. A., and Christiano, A. M., Hair follicle stem cells. J. Investig. Dermatol. Symp. Proc., 8, 28-38 (2003).

Lee, J. H., Lee, E. J., Lee, C. H., Park, J. H., Han, J. Y., and Lim, J. M., Requirement of leukemia inhibitory factor for establishing and maintaining embryonic stem cells in mice. Fertil. Steril., 92, 1133-1140 (2009).

Lee, J.-S., Bae, G.-Y., Lee, M.-O., and Cha, H.-J., Oncogenic challenges in stem cells and the link to cancer initiation. Arch. Pharm. Res., 35, 235-244 (2012).

Lendahl, U., Zimmerman, L. B., and McKay, R. D., CNS stem cells express a new class of intermediate filament protein. Cell, 60, 585-595 (1990).

Longoni, B. and Mosca, F., Stem cell-based immunomodulation in type 1 diabetes: beyond the regenerative approach. Curr. Pharm. Des., 17, 3229-3242 (2011).

Majumder, M. A. and Cohen, C. B., Research ethics. The NIH draft guidelines on human stem cell research. Science, 324, 1648-1649 (2009).

Murry, C. E. and Keller, G., Differentiation of embryonic stem cells to clinically relevant populations: lessons from embryonic development. Cell, 132, 661-680 (2008).

Okita, K., Ichisaka, T., and Yamanaka, S., Generation of germline-competent induced pluripotent stem cells. Nature, 448, 313-317 (2007).

Okita, K., Nagata, N., and Yamanaka, S., Immunogenicity of induced pluripotent stem cells. Circ. Res., 109, 720-721 (2011).
Orkin, S. H. and Zon, L. I., Hematopoiesis: an evolving paradigm for stem cell biology. Cell, 132, 631-644 (2008).

Pittenger, M. F., Mackay, A. M., Beck, S. C., Jaiswal, R. K., Douglas, R., Mosca, J. D., Moorman, M. A., Simonetti, D. W., Craig, S., and Marshak, D. R., Multilineage potential of adult human mesenchymal stem cells. Science, 284, 143-147 (1999).

Rando, T. A., The immortal strand hypothesis: segregation and reconstruction. Cell, 129, 1239-1243 (2007).

Sareen, D., McMillan, E., Ebert, A. D., Shelley, B. C., Johnson, J. A., Meisner, L. F., and Svendsen, C. N., Chromosome 7 and 19 trisomy in cultured human neural progenitor cells. PLoS One, 4, e7630 (2009).

Shin, J. W., Park, S. H., Kang, Y. G., and Shin, J.-W., Potential of engineering methodologies for the application to pharmaceutical research. Arch. Pharm. Res., 35, 299309 (2012).

Song, M., Paul, S., Lim, H., Dayem, A. A., and Cho, S.-G., Induced pluripotent stem cell research: a revolutionary approach to face the challenges in drug screening. Arch. Pharm. Res., 35, 245-260 (2012).

Stump, M. M., Jordan, G. L., Jr., Debakey, M. E., and Halpert, B., Endothelium grown from circulating blood on isolated intravascular dacron hub. Am. J. Pathol., 43, 361-367 (1963).

Tarte, K., Gaillard, J., Lataillade, J. J., Fouillard, L., Becker, M., Mossafa, H., Tchirkov, A., Rouard, H., Henry, C., Splingard, M., Dulong, J., Monnier, D., Gourmelon, P., Gorin, N. C., and Sensebe, L., Clinical-grade production of human mesenchymal stromal cells: occurrence of aneuploidy without transformation. Blood, 115, 1549-1553 (2010).

Yi, T. and Song, S. U., Immunomodulatory properties of mesenchymal stem cells and their therapeutic applications. Arch. Pharm. Res., 35, 213-221 (2012).

Yu, J., Vodyanik, M. A., Smuga-Otto, K., Antosiewicz-Bourget, J., Frane, J. L., Tian, S., Nie, J., Jonsdottir, G. A., Ruotti, V., Stewart, R., Slukvin, I. I., and Thomson, J. A., Induced pluripotent stem cell lines derived from human somatic cells. Science, 318, 1917-1920 (2007).

Zhou, X.-L., Sullivan, G. J., Sun, P., and Park, I.-H., Humanized murine model for HBV and HCV using human induced pluripotent stem cells. Arch. Pharm. Res., 35, 261-269 (2012). 